\title{
PRONET: Proactive Context-Aware Support for Mobility in Heterogeneous Access Networks
}

\author{
Karan Mitra \\ Caulfield School of Information Technology \\ 900 Dandenong Road, Caulfield East \\ Monash University, Australia \\ Karan.Mitra@infotech.monash.edu.au
}

\author{
Arkady Zaslavsky, Christer Åhlund \\ Luleå University of Technology \\ Division of Mobile Networking and Computing \\ SE-931 87 Skellefteå, Sweden \\ \{arkady.zaslavsky, christer.ahlund\}@1tu.se
}

\begin{abstract}
This paper presents a blueprint for proactive contextaware mobility support architecture for heterogeneous access networks called PRONET. In particular, we leverage upon the principles of cognitive networking to support proactive contextawareness for user-centric application adaptation via quality-ofexperience (QoE) provisioning. Our proposed architecture is built upon Port-based Multi-homed Mobile IPv6 (PM-MIPv6) solution to support several applications via path diversity. In this paper our contributions are two-fold. Firstly, we identify and present gaps in our research domain related to mobility, QoE, cognitive networks and cross-layer design. We then present our architecture for providing seamless mobility in heterogeneous access networks. Currently, we are in the process of collecting results via our test bed and prototype implementation for $\mathbf{8 0 2 . 1 1 \mathrm { g }}$ and HSDPA wireless networks.
\end{abstract}

Keywords-multihoming; quality of service (QoS); quality of experience (QoE); cognitive networks; cross-layer design

\section{INTRODUCTION}

Over the past decade we have witnessed a tremendous growth in the area of mobile and wireless computing. In order to support seamless application adaption, mobility solutions supporting multi-homing such as Multi-homed Mobile IP [1] are crucial. However, most of the proposals consider only one path at a time through which all the traffic needs to traverse. For example, consider a case of multi-mode terminal (MN) having WLAN and HSDPA interfaces. Here, all the traffic will be directed through one of these interfaces rather than using both the network interfaces simultaneously. This technique is inefficient as the other network interface remains idle. We argue that the unused interface should be used concurrently to support several applications via path-diversity. Path diversity can be used to build resilient networks which can lead to efficient flow-assignment and fail-over mechanisms. Thus, we consider Port-based Multi-homed Mobile IPv6 (PM-MIPv6) [2] through which a MN can roam between several wireless networks using several applications simultaneously, divided/shared among multiple access technologies.

To satisfy quality of service (QoS) requirements in terms of the user, quality of experience (QoE) [3] is an important factor to be considered. QoE can be measured by evaluating the user's responses directly by a number of user studies. However, it is a time consuming, error-prone and an expensive method.
Thus, several speech quality assessment techniques such as PESQ, ITU-T E-Model and pseudo-subjective quality assessment (PSQA) were proposed recently to map the objective quality onto the subjective score such as the Mean Opinion Score (MOS). We consider the ITU-T E-Model [4] as it is the most widely used method and can help in assessing the quality of VoIP session in real-time. While these models have been used extensively in the research literature, they have not been studied carefully in the light of the time-varying characteristics of wireless networks. Moreover, we believe that if we can obtain and leverage upon the statistical information using the aforementioned speech prediction models, proactive measures can be taken to satisfy the QoE of users using cognitive networking approach. In order to efficiently adapt to the QoE observed by the user, cross-layer and cognitive design methodology can be quite useful in the context of PM-MIPv6 [2], provided it is applied carefully. In [5], a cognitive network is defined as a network which is able to perceive and sense the current network conditions and then plan, decide and act on those conditions to achieve end-to-end goals. However, there is a dearth of research literature dealing with cognitive and crosslayer optimization for multi-homed solutions especially where the learning and reasoning is done in a distributed manner.

Significant amount of work has been done in the field of context-awareness. By using context-aware techniques such as [6], user-centric behavior and its effects on mobility protocols can be studied. Techniques such as multi-sensor data fusion along with learning paradigms can assist in developing robust networking protocols to enhance the end-to-end QoE of the user. To achieve seamless user experience in future wireless networks, we enlist the following challenges: a) determine the mapping between QoS and QoE; b) end-to-end QoE prediction focusing on user location and his/her movement patterns; c) proactive path selection based on path diversity; and d) understanding the effects of other nodes in the network on QoE provisioning in multi-homed solutions.

\section{PRONET ARCHITECTURE}

We propose that a $\mathrm{MN}$ should provide opportunistic network connectivity options to the user by simultaneously connecting to several network technologies concurrently, in a proactive manner. Fig. 1 shows our targeted scenario. As shown in this figure, a MN using PM-MIPv6 [2] can roam between several 
wireless networks using several applications simultaneously. For example, a MN can leverage upon the WLAN network to support voice over IP (VoIP) and FTP while HSDPA can be used for HTTP, concurrently. This scenario can be quite challenging when the $\mathrm{MN}$ is moving, particularly at high speeds. To provide seamless mobility in such situations, proactive QoE determination and provisioning techniques along with robust opportunistic network selection methods will be required as mentioned in the previous section. Fig. 2 shows our proposed approach to tackle the aforementioned challenges based on proactive context-awareness and PM-MIPv6. We first define the mapping between QoS and QoE. We then try to predict the QoE in advance and finally, we select the best available path based on prior learned experiences by considering other nodes in the networks.

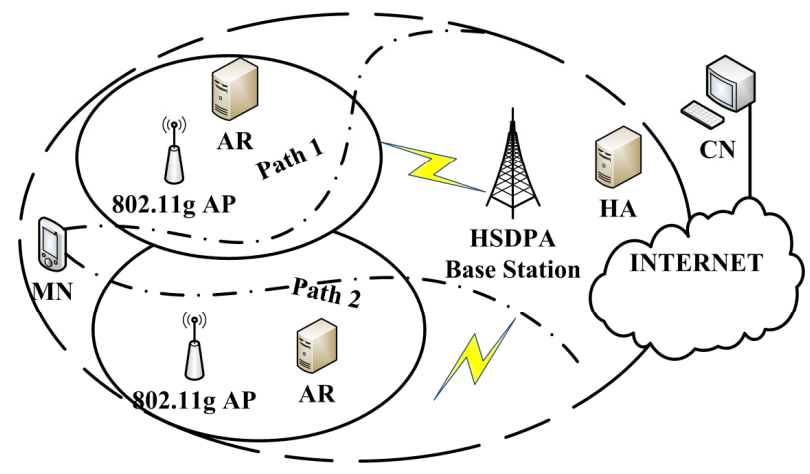

Figure 1. Opportunistic access network selection using PM-MIPv6.

\section{A. Proactive Context-awareness for Mobility Support in Heterogeneous Access Networks}

We consider the Context Spaces Model (CSM) [6] to make sense of the context information and then determine the situations. These situations can be based on the overall QoE and user movement patterns to determine the best network for running applications. Currently, the CSM does not include situation prediction. We are extending this model to determine, learn and predict the situations in a distributed manner using Reinforcement Learning (RL) [7]. For example, situations related to network load on the AP and QoS observed by the $\mathrm{MN}$ can be fused together to determine QoE efficiently. Fig. 3 presents the process of learning and adaptation of situations based on RL [7]. In this case, current situations can be determined based on the user's movement patterns, his/her perceived QoE, etc. and then based on learning and prediction algorithms, future situations can be determined. Consequently, efficient algorithms can be developed for path selection.

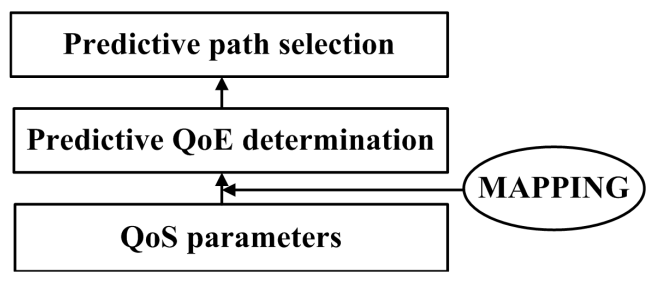

Figure 2. Our proposed approach.

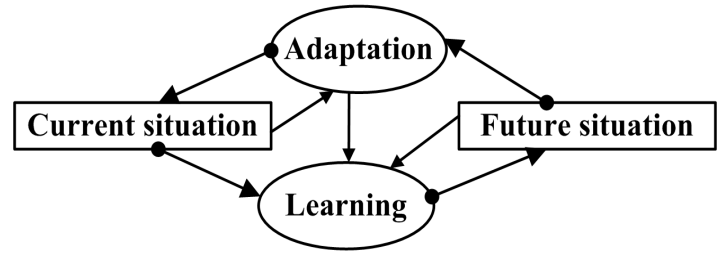

Figure 3. Situation learning and adaptation using RL.

\section{B. Context-aware QoS to QoE Mapping and Prediction}

As mentioned previously, there is a dearth of research literature looking into time-varying characteristics of wireless networks w.r.t QoE provisioning. At the moment we are in the midst of finding out the QoS and QoE relationship for several voice codecs. Our first goal is to determine and learn the most suitable situation(s) based on the QoE observed by user in time-varying network conditions. For learning and prediction we consider statistical learning techniques such as Continuoustime Hidden Markov Model (CT-HMM). In this model, we are using several parameters from various layers of the protocol stack such as: delay, jitter, packet loss, signal to noise ratio (SNR) and location to predict the overall mean opinion score (MOS) for a particular situation. We have also developed an experimental test bed and a prototype and are in the process of collecting results for $802.11 \mathrm{~g}$ and HSDPA wireless networks.

\section{Predictive path selection using cross-layer design}

Once the MN determines the QoE in advance for the available network paths, our MN should proactively adjust to the underlying network conditions at the right time. We consider IEEE 802.21 standard as an enabler for efficient network selection build a cross-layer mechanism for proactive network selection.

\section{REFERENCES}

[1] C. Ahlund, R. Brannstrom, and A. Zaslavsky, "M-MIP: extended Mobile IP to maintain multiple connections to overlapping wireless access networks," Lecture Notes in Computer Science, vol. 3420, p. 204, 2005.

[2] C. Ahlund, R. Brannstrom, K. Andersson, and O. Tjernstrom, "Portbased Multihomed Mobile IPv6 for Heterogeneous Networks," in Local Computer Networks, Proceedings 2006 31st IEEE Conference on, 2006, pp. 567-568.

[3] A. P. C. da Silva, M. Varela, E. de Souza e Silva, R. M. M. Leão, and G. Rubino, "Quality assessment of interactive voice applications," Computer Networks, vol. 52, pp. 1179-1192, 2008.

[4] ITU-T Recommendation G.107, The E-Model, a Computational Model for Use in Transmission Planning, 2000.

[5] R. W. Thomas, L. A. DaSilva, and A. B. MacKenzie, "Cognitive networks," in New Frontiers in Dynamic Spectrum Access Networks, 2005. DySPAN 2005. 2005 First IEEE International Symposium on, 2005, pp. 352-360.

[6] A. Padovitz, S. W. Loke, and A. Zaslavsky, "Towards a theory of context spaces," in Pervasive Computing and Communications Workshops, 2004. Proceedings of the Second IEEE Annual Conference on, 2004, pp. 38-42.

[7] G. T. Dietterich and P. Langley, "Machine learning for cognitive networks: Technology assessment and research challenges," in Cognitive Networks: Towards Self-Aware Networks, Q. H. Mahmoud, Ed.: John Wiley \& Sons Ltd. , 2007. 